\title{
Shunt Testing In Vivo: Illustration of Partially Obstructed Ventricular Catheter by In-Growing Choroid Plexus
}

\author{
Virginia Levrini ${ }^{1}$, Zofia Czosnyka ${ }^{1}$, Indu Lawes ${ }^{1}$, Angelos G. Kolias ${ }^{2}$, Richard Mannion ${ }^{1}$ \\ 1. Neurosurgery, Addenbrooke's Hospital, Cambridge University Hospitals NHS Foundation Trust, Cambridge, GBR 2. \\ Clinical Neurosciences, Addenbrooke's Hospital and University of Cambridge, Cambridge, GBR
}

Corresponding author: Virginia Levrini, virginialevrini@gmail.com

\begin{abstract}
Assessing shunt function in vivo presents a diagnostic challenge. Infusion studies can be a cost-effective and minimally invasive aid in the assessment of shunt function in vivo. We describe a case of a patient who after a foramen magnum decompression for type I Chiari malformation developed bilateral posterior fossa subdural hygromas and mild hydrocephalus, eventually necessitating insertion of a ventriculoperitoneal shunt. The patient returned with symptoms that were concerning for infection of the shunt. A bedside infusion study helped confirm that the ventricular catheter was partially obstructed by in-growing choroid plexus, but also that the shunt was no longer necessary. Partial blockage due to in-growing choroid plexus was confirmed during surgery to remove the shunt. We discuss the behaviour of in-growing choroid plexus and how partial obstruction can be detected with the use of an infusion study, as well as how this compares to the pattern observed in complete shunt obstruction. The benefits of using infusion studies in the assessment of shunt function are also explored.
\end{abstract}

Categories: Neurosurgery

Keywords: cerebrospinal fluid, hydrocephalus, chiari malformation, ventriculoperitoneal shunt, neurosurgery

\section{Introduction}

Obstructed, underdraining or overdraining shunts may disturb optimal management of hydrocephalus. Proper evaluation of shunt function in vivo often presents a challenge. Different techniques are used, including microflowmetry, ultrasonography or MRI techniques [1-3]. All of them require specialised equipment and expertise for interpretation of the results. Moreover, most of these methods rely on detection of spontaneous flow of cerebrospinal fluid (CSF) through the shunt, which may not be present at the time of testing even in a patent shunt. Reliable shunt testing in vivo is important as clinical symptoms and brain imaging data may be incongruous. In the case of the shunt functioning well, it spares unnecessary revision.

Received 07/02/2020

Review began $07 / 12 / 2020$ Review ended 07/13/2020 Published 07/19/2020

๑) Copyright 2020

Levrini et al. This is an open access article distributed under the terms of the Creative Commons Attribution License CC-BY 4.0., which permits unrestricted use, distribution, and reproduction in any medium, provided the original author and source are credited.
Infusion tests to assess shunt function allow quick, objective assessment of shunt function with forced accelerated flow of fluid through the shunt. Two $25 \mathrm{G}$ needles are inserted transcutaneously into the shunt pre-chamber using sterile preparation and careful skin cleaning [4]. Through one needle Hartmann's fluid is infused and pressure recording is performed through the second needle. In the case of obstruction of the intraventricular catheter, three findings are specific [5]. Firstly, baseline recorded CSF pressure is without any or with very small pulsations synchronised to the heart rate. Secondly, after starting the infusion, CSF pressure increases precipitously (within 10-20 seconds) to a value slightly above the shunt opening pressure. Thirdly, if the shunt has a subcutaneous chamber siphon control device, compression of the chamber during infusion blocks flow through the shunt and recorded pressure increases to a high value immediately (usually above $50 \mathrm{~mm}$ Hg with a delay of only a few seconds).

We present a case in which ventricular catheter blockage was detected with an infusion test and the finding was confirmed during revision surgery.

\section{Case Presentation}

A 37-year-old female patient with a symptomatic type I Chiari malformation underwent foramen magnum decompression in our department. The operation was uneventful and she was discharged home two days post-operatively.

She presented two months later with a two-week history of headaches exacerbated by lying flat. A CT head showed small bilateral posterior fossa subdural hygromas and mild hydrocephalus (Figure 1). As her symptoms progressed, she underwent posterior fossa re-exploration where the craniectomy was extended with two new burr holes placed laterally on both sides, the dura was opened and the CSF collections were drained. Some of the CSF was collected and as the microscopy demonstrated CSF pleocytosis (polymorphs 


\section{Cureus}

[PMNs] $156 \times 10^{6} / \mathrm{L}$, lymphocytes $24 \times 10^{6} / \mathrm{L}$, red blood cells $32 \times 10^{6} / \mathrm{L}$ ), she was started on IV ceftriaxone pending culture results, which later returned as no significant growth. Her headache gradually subsided over the next two days. Three days post-operatively, she developed a wound leak, which persisted despite resuturing.
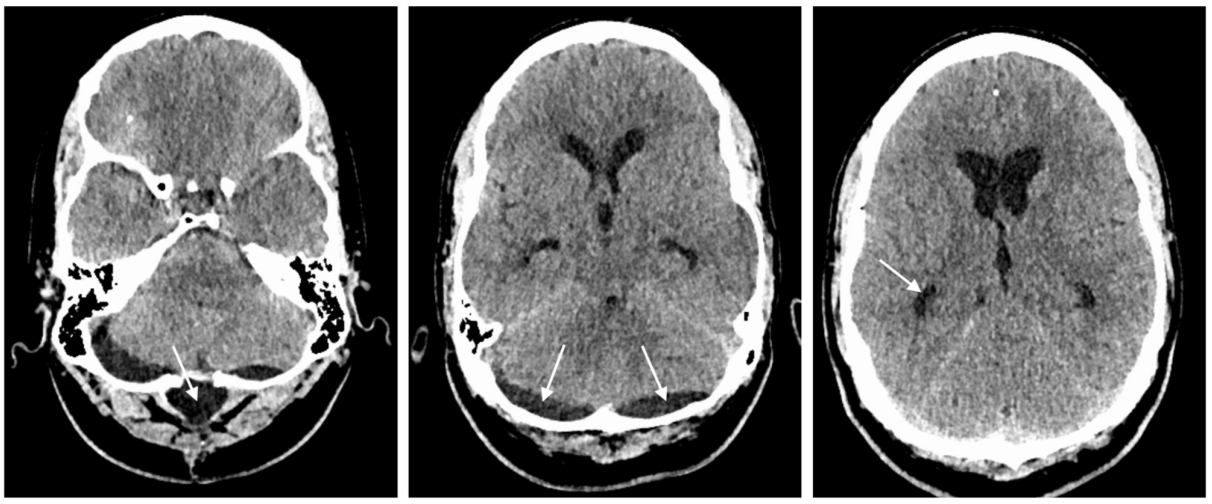

FIGURE 1: CT head two months following foramen magnum decompression.

CT head demonstrating small pseudomeningocele in relation to foramen magnum decompression (arrow in left image), bilateral posterior fossa subdural hygromas (arrows in middle image) and mild hydrocephalus with dilated temporal horns (arrow in right image).

A repeat CT head showed resolution of the hygromas and an unchanged degree of mild hydrocephalus; in view of this and the persistent leak, a ventriculoperitoneal shunt (VPS) was offered to the patient (Figure 2). A right parietal VPS was inserted with a programmable valve (small Strata II valve (Medtronic, Dublin, Ireland) set at 1.5) without complications. No further wound leaks were noted and the patient was discharged home the following day.
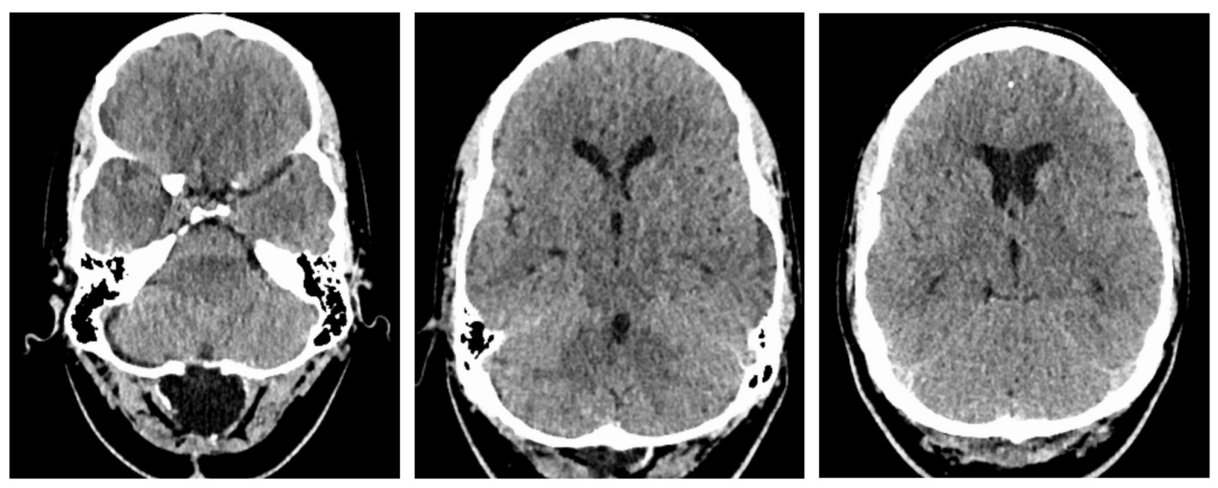

FIGURE 2: CT head after posterior fossa re-exploration surgery.

Repeat CT head demonstrating almost complete resolution of the posterior fossa subdural hygromas after surgical drainage and mild hydrocephalus.

The patient represented to the emergency department one month later with fever, headaches, neck pain, nausea and vomiting. Neurological examination revealed mild neck stiffness but no focal neurological signs. All incisions looked healthy. A CT head and X-rays of the shunt showed decompressed ventricles and continuity of the shunt system, respectively. The shunt pre-chamber was accessed with a $25 \mathrm{G}$ butterfly needle, but no CSF could be aspirated. A small pseudomeningocele was tapped, and microscopy showed raised lymphocytes $\left(570 \times 10^{6} / \mathrm{L}\right)$ but normal PMNs $\left(6 \times 10^{6} / \mathrm{L}\right)$ and no organisms on Gram stain. Her Creactive protein (CRP) was normal. She was started on a course of intravenous ceftazidime and vancomycin and a course of aciclovir. She was kept under close neurological observation. She continued to have fever intermittently over the next few days but remained clinically well. Hence, she was reviewed by the infectious diseases team who could not identify a source of the fever but concluded that a VPS infection could not be excluded. As the patient was initially reluctant to undergo another operation, an infusion study was organised to assess her CSF circulation. Before starting the infusion, CSF was aspirated from the shunt prechamber with microscopy revealing $4 \times 10^{6} / \mathrm{L}$ PMNs and $8 \times 10^{6} / \mathrm{L}$ lymphocytes. The infusion study results 


\section{Cureus}

revealed partial obstruction of the ventricular catheter but normal baseline intracranial pressure (ICP), suggesting that the patient was not shunt-dependent (Figure 3).

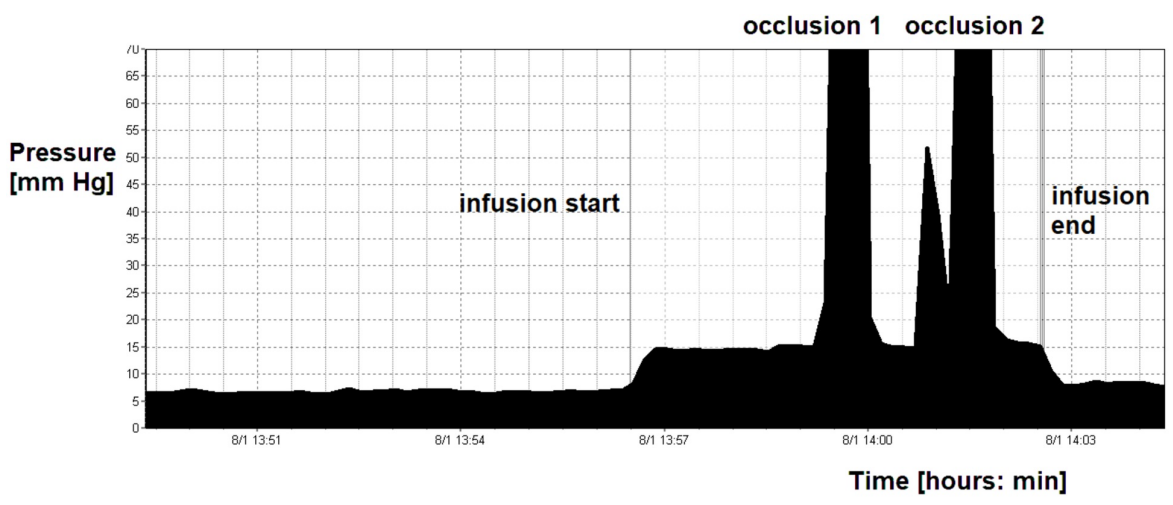

\section{FIGURE 3: Recording of pre-chamber pressure during infusion test.}

During the first eight minutes, the pressure was around $7 \mathrm{~mm} \mathrm{Hg}$ with minimal but detectable pulsations (around $0.1 \mathrm{~mm} \mathrm{Hg}$ ). During infusion, pulsations disappeared and pressure very quickly reached $15 \mathrm{~mm} \mathrm{Hg}$, which is in agreement for Strata valve working at 1.5. Further two compressions of the siphon control device were performed. During compression, pressure increased to above $150 \mathrm{~mm} \mathrm{Hg}$ without delay. After end of infusion, pressure decreased to pre-infusion level and minimal pulsation re-appeared. Remarkably, after insertion of needles into the shunt pre-chamber, cerebrospinal fluid could be aspirated fluently.

The option of removing the shunt was discussed with the patient, explaining the small possibility that she might need another VPS at a later date. The patient agreed to proceed and the shunt was removed without complications. When the ventricular catheter was removed, it was noted that choroid plexus was adherent to the ventricular catheter tip (Figure 4). The tip was cultured, with no growth found after two days incubation. The patient was well post-operatively and was discharged home after 24 hours. She remains well at four-month follow-up.

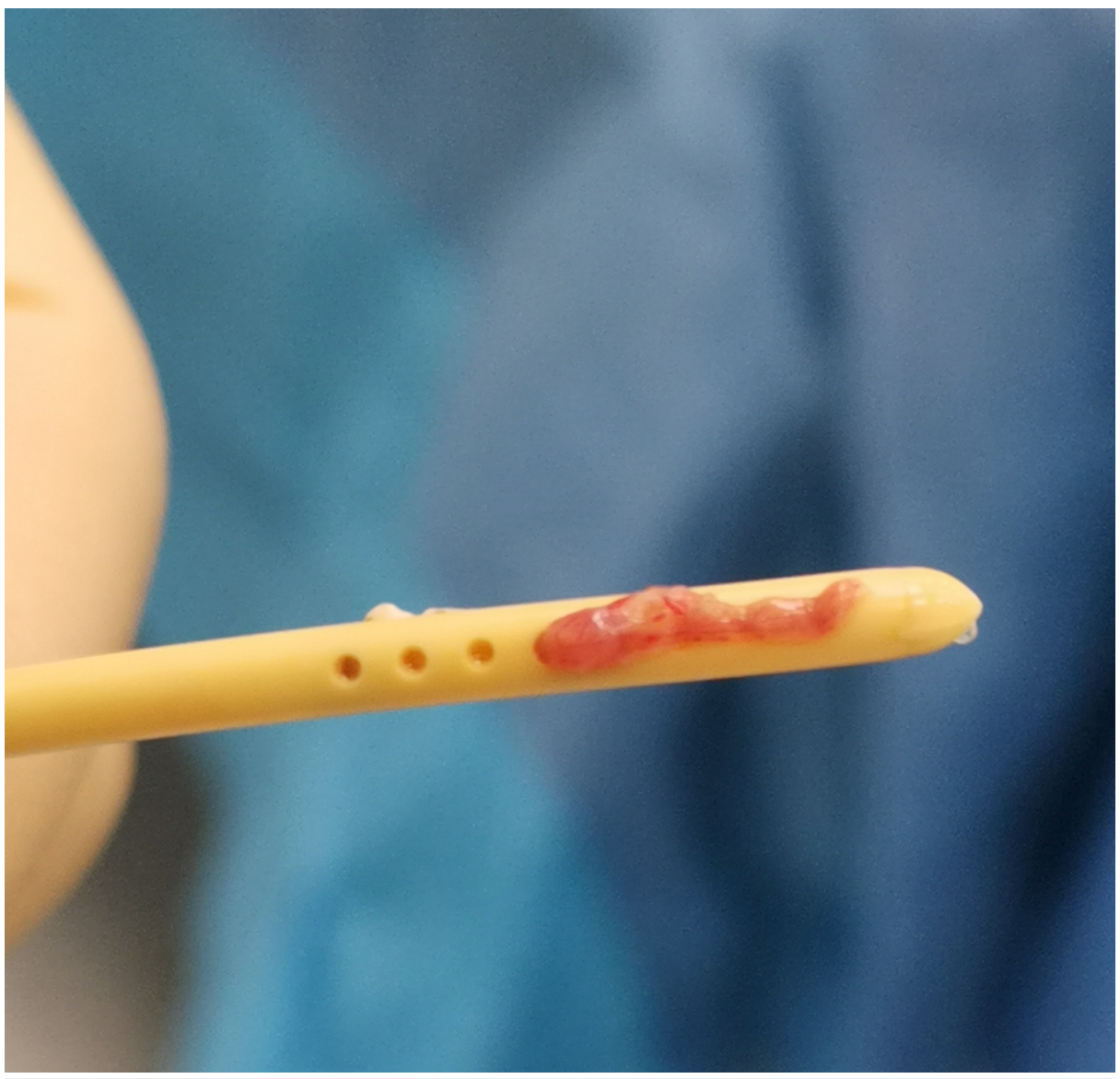

FIGURE 4: Photo of explanted ventricular catheter. 


\section{Discussion}

A number of important lessons can be learnt from this case. First, shunt blockage at the ventricular end can be detected with relative ease. In this case, the intraoperative findings confirmed the conclusion drawn from the infusion study. An in-growing choroid plexus does not necessarily completely block the shunt. Flow of CSF can be only partially blocked; therefore, aspiration of CSF from the shunt pre-chamber is possible. Choroid plexuses behave as a "water weed" in a river, aligning in a direction and not necessarily fully blocking CSF flow. Additionally, a pulse waveform at baseline can be seen; however, this is usually very small and disappears during infusion. When the pressure in the shunt pre-chamber increases during infusion, the longitudinal orientation of the in-growing plexus is disturbed and it firmly blocks the communication between the shunt and cerebral ventricles (Figure 5). The pulse amplitude disappears and the shunt starts to behave as if there is a solid ventricular block. Therefore, during occlusion, pressure in the pre-chamber rises to very high values without delay. In contrast, when the intraventricular end is completely blocked, no pulse waveform of ICP can be seen and it is impossible to aspirate any CSF from the shunt pre-chamber. Other findings are the same as in partial blockage by in-growing choroid plexus. Shunt testing in vivo is helpful in making the right decision in complex cases. In this particular case, the improperly working shunt was removed as the disturbed CSF circulation appeared to be transient based on the finding of normal baseline ICP.

A.

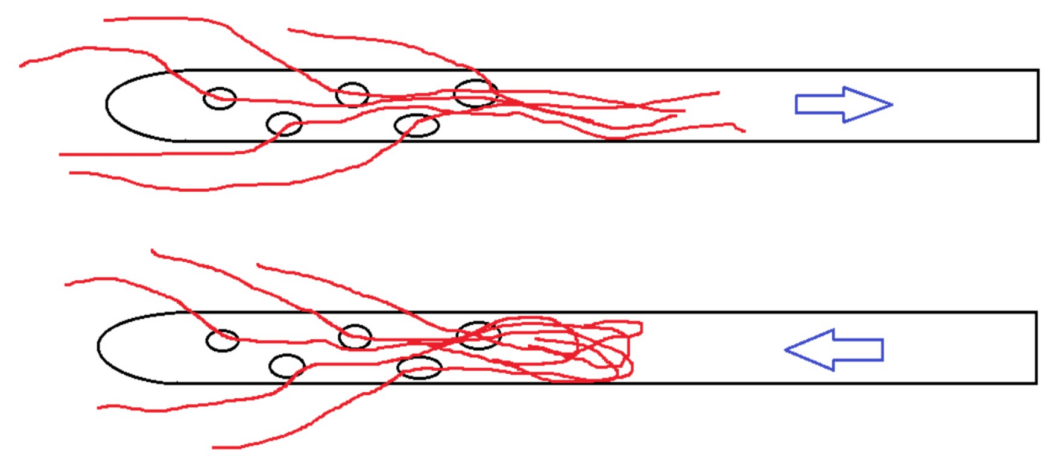

\section{FIGURE 5: Illustration of "water weed" behaviour.}

(A) When choroid plexus in-grows into ventricular catheter, flow towards the shunt is partially obstructed, but possible. Aspiration of cerebrospinal fluid is possible, pulse amplitude in measured pressure is usually damped. (B) During infusion pressure in the pre-chamber increases, choroid plexus disrupts communication of pre-chamber with ventricles. Pulse amplitude in recording disappears. During occlusion of the siphon control device, shunt behaves as proximally blocked.

The second lesson is about decision to shunt. Shunts for hydrocephalus principally facilitate poor CSF circulation. With a decision to shunt made on the basis of clinical symptoms and brain imaging, the relevant symptoms improve after shunting in approximately $60 \%$ to $90 \%$ of cases. However, in $10 \%$ to $40 \%$ of cases, the improvement is partial or absent altogether [6]. Therefore, it is logical, especially considering the low invasiveness of an infusion test, to confirm if the CSF circulation is indeed impaired before first-time shunting in equivocal cases. In the described case, such an investigation was not initially conducted because the patient had a persistent CSF leak through the recently re-opened wound, making the decision to insert a shunt straightforward.

Finally, shunt testing in vivo can also prevent unnecessary revisions, if the shunt is found to work well during infusion test. Our recent study suggests that in approximately $50 \%$ of the tests in patients with a shunt in situ, the shunt system is patent [4]. Appropriate management will then depend on the specifics of each case but may include a change in the shunt setting, implantation of an anti-siphon device or treatment of symptoms that are due to another condition. As well as avoiding the risks associated with revision, the average financial gain by unnecessary revisions in our hospital is around one million pounds per year [4]. Infusion tests are safe. The risk of infection or other complications is less than $0.6 \%$.

\section{Conclusions}

We describe a case of a partially obstructed ventricular catheter by in-growing choroid plexus. Partial blockage was ascertained with a bedside infusion study and the results confirmed during surgery. As illustrated by this patient's case, it can be difficult to detect partial blockage using clinical picture and shunt 
tap only. Therefore, we discuss the expected results during infusion study in the case of partial blockage and the underlying mechanism in the case of an in-growing choroid plexus. Shunt infusion studies can assist in decision making for patients presenting with suspected shunt malfunction.

\section{Additional Information \\ Disclosures}

Human subjects: Consent was obtained by all participants in this study. Conflicts of interest: In compliance with the ICMJE uniform disclosure form, all authors declare the following: Payment/services info: All authors have declared that no financial support was received from any organization for the submitted work. Financial relationships: All authors have declared that they have no financial relationships at present or within the previous three years with any organizations that might have an interest in the submitted work. Other relationships: All authors have declared that there are no other relationships or activities that could appear to have influenced the submitted work.

\section{Acknowledgements}

We thank the patient for providing informed consent for this case report. This case report was written according to the CARE guidance.

\section{References}

1. Miyake H, Ohta T, Kajimoto Y, Nagao K: New concept for the pressure setting of a programmable pressure valve and measurement of in vivo shunt flow performed using microflow meter. J Neurosurg. 2000, 92:181187. 10.3171/jns.2000.92.1.0181

2. Lam KW, Drake JM, Cobbold RS: Noninvasive cerebrospinal fluid shunt flow measurement by Doppler ultrasound using ultrasonically excited bubbles: a feasibility study. Ultrasound Med Biol. 1999, 25:371-389. 10.1016/S0301-5629(98)00164-1

3. Blitz AM, Huynh PP, Bonham LW, et al.: High-resolution MRI for evaluation of ventriculostomy tubes: assessment of positioning and proximal patency. AJNR Am J Neuroradiol. 2020, 41:57-63.

4. Lalou AD, Czosnyka M, Garnett MR, et al.: Shunt infusion studies: impact on patient outcome, including health economics. Acta Neurochir. 2020, 162:1019-1031. 10.1007/s00701-020-04212-0

5. Czosnyka ZH, Sinha R, Morgan JA, et al.: Shunt testing in vivo: observational study of problems with ventricular catheter. Intracranial Pressure and Brain Monitoring XV. Ang BT (ed): Springer, Cham; 2016. 122:353-356. 10.1007/978-3-319-22533-3_69

6. Saehle T, Farahmand D, Eide PK, Tisell M, Wikkelso C: A randomized controlled dual-center trial on shunt complications in idiopathic normal-pressure hydrocephalus treated with gradually reduced or fixed pressure valve settings. J Neurosurg. 2014, 121:1257-1263. 10.3171/2014.7.JNS14283 